\title{
Use of pioglitazone in the treatment of diabetes: effect on cardiovascular risk
}

\author{
This article was published in the following Dove Press journal: \\ Vascular Health and Risk Management \\ 24 July 2013 \\ Number of times this article has been viewed
}

\section{Cong Zou' \\ Honglin $\mathrm{Hu}^{2}$ \\ 'Department of Endocrinology, Fourth Affiliated Hospital of Nanchang University, Nanchang, People's Republic of China; ${ }^{2}$ Department of Urology, Second Affiliated Hospital of Nanchang University, Nanchang, People's Republic of China}

Correspondence: Honglin $\mathrm{Hu}$ Department of Urology, Second Affiliated Hospital of Nanchang University, I Minde Road, Nanchang, Jiangxi 330006,

People's Republic of China

Tel +86 79l 86300640

Email honglinhu@I26.com
Abstract: Pioglitazone and other thiazolidinediones (TZDs) initially showed great promise as unique receptor-mediated oral therapy for type 2 diabetes, but a host of serious side effects, primarily cardiovascular, have limited their utility. It is crucial at this point to perform a riskbenefit analysis to determine what role pioglitazone should play in our current treatment of type 2 diabetes and where the future of this class of drugs is headed. This review provides a comprehensive overview of the present literature. Clinical data currently available indicate that pioglitazone is an effective and generally well-tolerated treatment option for use in patients with type 2 diabetes. Pioglitazone can still reduce adverse cardiovascular risk.

Keywords: pioglitazone, diabetes, cardiovascular risk

\section{Introduction}

Thiazolidinediones (TZDs) are synthetic exogenous agonists of peroxisome proliferator-activated receptor- $\gamma(\operatorname{PPAR} \gamma)$ and are used in the treatment of type 2 diabetes mellitus. Currently, two TZDs, rosiglitazone and pioglitazone, are available, although rosiglitazone is being withdrawn from the market in Europe and its use is restricted in the US, due to concerns about the increase in prevalence of myocardial infarction. TZDs increase insulin sensitivity, reduce blood glucose and hemoglobin A1c levels, inhibit adipose-tissue lipolysis, decrease microalbuminuria, inhibit inflammation, and reduce blood pressure in experimental animals and in TZD-treated patients. ${ }^{1,2}$

The results of the Rosiglitazone Evaluated for Cardiac Outcomes and Regulation of Glycemia in Diabetes (RECORD) trial, which was once considered to be the strongest evidence evaluating the cardiovascular safety of rosiglitazone, showed no significant increase in these events with rosiglitazone compared to metformin or sulfonylurea in $2009 .{ }^{3}$ On the other hand, a meta-analysis of 19 randomized controlled trials with pioglitazone found a statistically significant reduction in the composite outcome of nonfatal myocardial infarction, stroke, and all-cause mortality. ${ }^{4}$ Thus, the cardiovascular risk data for rosiglitazone and benefits of pioglitazone are less than conclusive, and an intriguing disparity between the drugs has emerged, suggesting an intraclass variation in TZD effects. Moreover, another meta-analysis compared the cardiovascular risks of rosiglitazone and pioglitazone based on a total of eight retrospective cohort studies. The risk of all three outcomes - myocardial infarction, heart failure, and all-cause mortality - was higher in the rosiglitazone group than in the pioglitazone group.

In 2007, a meta-analysis based on 42 randomized trials suggested that TZDs increased the risk of myocardial infarction and cardiovascular deaths. ${ }^{5}$ It was 
believed that the incidence of cardiac defects was lower with pioglitazone, but subsequent trials indicated no difference in complications between pioglitazone and rosiglitazone..$^{6,7}$

The PROACTIVE (Prospective Pioglitazone Clinical Trial in Macrovascular Events) study conducted on diabetic patients indicated $11 \%$ increased chances of congestive heart failure in pioglitazone-treated patients compared to placebotreated patients. ${ }^{8}$ Similarly, trials involving rosiglitazone also indicated complications of heart failure. ${ }^{9}$ These studies presumed that the chance of heart failure is more prevalent in diabetics who have a history of cardiac problems..$^{7-9}$

The PROACTIVE trial studied more than 5,000 patients with diabetes at high risk for macrovascular complications, and reported that treatment with pioglitazone produced a nonsignificant reduced risk for coronary and peripheral vascular events. ${ }^{10}$ For a secondary end point, a composite of death, myocardial infarction, or stroke, a statistically significant benefit was observed. However, pioglitazone increased the incidence of congestive heart failure (although not of mortality associated with heart failure). Moreover, it was unclear whether findings among the patients in the PROACTIVE study could be extrapolated to lower-risk populations of diabetic patients without established vascular disease.

The PROACTIVE study had some methodological and analytical limitations. The time period of the study may have been too short, and the patients were nearly all white and from European countries. Moreover, the patients were at a very late stage of the disease process.

However, larger trials involving more patients concluded that the chances of heart failure with TZD therapy is equal in both diabetic and nondiabetic patients. ${ }^{11}$ There are uncertainties regarding the cardiovascular toxic effects of TZDs. According to a retrospective cohort study conducted on more than 16,000 patients, although TZDs may increase peripheral edema, a symptom of heart failure, they did not increase mortality and may actually decrease it. ${ }^{12}$

Considering the complex cardiovascular action of TZDs and lack of sufficient data to support the clinical finding, this review was planned to evaluate the influence of pioglitazone on cardiovascular outcomes in patients with type 2 diabetes mellitus.

\section{Pioglitazone and progression of coronary atherosclerosis}

Atherosclerosis in patients with diabetes is particularly aggressive, characterized by higher cardiovascular event rates and a greater severity of coronary obstructive disease. ${ }^{13-16}$ Cardiovascular disease represents the ultimate cause of death in approximately $75 \%$ of patients with diabetes. ${ }^{17}$ The PERISCOPE (Pioglitazone Effect on Regression of Intravascular Sonographic Coronary Obstruction Prospective Evaluation) trial $^{18}$ compared the effects of an insulin sensitizer, pioglitazone, with an insulin secretagogue, glimepiride, on the progression of coronary atherosclerosis in patients with type 2 diabetes, and found that least squares mean percentage atheroma volume increased $0.73 \%(95 \%$ confidence interval [CI] $0.33 \%-1.12 \%$ ) with glimepiride and decreased $0.16 \%$ (95\% CI $-0.57 \%$ to $0.25 \%)$ with pioglitazone $(P=0.002)$. An alternative analysis imputing values for noncompleters based on baseline characteristics showed an increase in percentage atheroma volume of $0.64 \%$ (95\% CI $0.23 \%-1.05 \%)$ for glimepiride and a decrease of $0.06 \%$ ( $-0.47 \%$ to $0.35 \%)$ for pioglitazone (between-group $P=0.02$ ). Mean (standard) baseline hemoglobin A1c levels were $7.4 \%(1.0 \%)$ in both groups and declined during treatment an average of $0.55 \%(95 \% \mathrm{CI}-0.68 \%$ to $-0.42 \%)$ with pioglitazone and $0.36 \%(95 \% \mathrm{CI}-0.48 \%$ to $-0.24 \%)$ with glimepiride (between-group $P=0.03$ ). In the pioglitazone group, compared with glimepiride, high-density lipoprotein levels increased $5.7 \mathrm{mg} / \mathrm{dL}(95 \%$ CI $4.4-7.0 \mathrm{mg} / \mathrm{dL}$, $16.0 \%$ ) vs $0.9 \mathrm{mg} / \mathrm{dL}$ (95\% CI -0.3 to $2.1 \mathrm{mg} / \mathrm{dL}, 4.1 \%$ ), and median triglyceride levels decreased $16.3 \mathrm{mg} / \mathrm{dL}(95 \%$ $\mathrm{CI}-27.7$ to $-11.0 \mathrm{mg} / \mathrm{dL}, 15.3 \%$ ) vs an increase of $3.3 \mathrm{mg} / \mathrm{dL}$ (95\% CI -10.7 to $11.7 \mathrm{mg} / \mathrm{dL}, 0.6 \%)(P<0.001$ for both comparisons). Median fasting insulin levels decreased with pioglitazone and increased with glimepiride $(P<0.001)$. The findings of the PERISCOPE study support the conclusion that treatment with the insulin-sensitizing TZD pioglitazone compared with glimepiride can prevent the progression of atherosclerosis in patients with type 2 diabetes during 18 months of treatment. Patients randomized to pioglitazone exhibited a lower rate of progression of coronary atherosclerosis across a wide array of prespecified and exploratory subgroups.

\section{Pioglitazone and urinary albumin excretion}

Microalbuminuria is another strong risk indicator for cardiovascular events, and has been suggested as a marker for patients with endothelial and renal dysfunction, particularly in patients with features of metabolic syndrome. ${ }^{19-21}$ In a multicenter, double-blind study, ${ }^{22}$ patients were randomized to receive either pioglitazone $15 \mathrm{mg}(\mathrm{n}=319)$ or metformin $850 \mathrm{mg}(\mathrm{n}=320)$ and up to $45 \mathrm{mg} /$ day and $2,550 \mathrm{mg} /$ day, respectively. Pioglitazone reduced the urinary albumin-tocreatinine ratio by $15 \%$ in combination with a sulfonylurea. In contrast, metformin as add-on therapy to a sulfonylurea 
increased urinary albumin-to-creatinine ratio by $2 \%$. These changes were not related to differences between groups in changes in blood pressure or in the use of agents acting on the renin-angiotensin system ( $44 \%$ in each group, primarily angiotensin-converting-enzyme inhibitors). Although the clinical significance of this is uncertain, improvement in a cardiovascular risk marker with pioglitazone treatment, which is of a similar order to that seen with angiotensinconverting-enzyme inhibitors, ${ }^{23}$ may be of value in this high-risk group. Because pioglitazone significantly improves dyslipidemia and urinary albumin-to-creatinine ratio, any improvements in cardiovascular risk factors may be demonstrated in ongoing pioglitazone outcome studies.

To determine whether pioglitazone affects urinary albumin excretion (UAE) or the number of urinary podocytes or both in type 2 diabetes patients with microalbuminuria, 28 patients with normotensive type 2 diabetes and microalbuminuria (18 men, ten women, mean age 52.5 years) and 30 age-matched normotensive controls (20 men, ten women, mean age 51.5 years) were included in a study. Urinary podocytes were detected by immunofluorescence with a monoclonal antibody against podocalyxin. Patients were randomly assigned to two groups: a pioglitazonetreatment group (30 mg/day, $\mathrm{n}=14$ ) and a placebo group $(n=14)$. Treatment was continued for 6 months. Podocytes were absent in the urine of healthy controls, but detected in 17 of 28 diabetic patients $(60.7 \%)$. UAE was reduced from $96.7 \pm 50.5 \mu \mathrm{g} /$ minute to $39.7 \pm 22.9 \mu \mathrm{g} /$ minute $(P<0.01)$ in the pioglitazone-treatment group, and the number of urinary podocytes was reduced from $0.9 \pm 1.0$ cells $/ \mathrm{mL}$ to $0.1 \pm 0.2$ cells $/ \mathrm{mL}(P<0.001)$. Neither UAE nor the number of urinary podocytes was affected in the placebo group. These data indicate that pioglitazone is effective for reducing UAE and podocyte injury in early stage diabetic nephropathy. ${ }^{24}$

\section{Pioglitazone and changes in lipid profiles}

The effects of lipids on cardiovascular disease are well known. Type 2 diabetes patients with dyslipidemia have a markedly increased risk of cardiovascular disease compared to nondiabetic patients with similar lipid levels. ${ }^{25}$ Diabetic dyslipidemia is characterized by reduced levels of highdensity lipid (HDL) cholesterol, elevated triglycerides, and a high proportion of smaller and denser low-density lipid (LDL) particles. ${ }^{26}$ Potential lipid-altering effects of oral antidiabetic agents, including those in the thiazolidinedione class, have also been evaluated in type 2 diabetes patients.
In a previous head-to-head trial, although both pioglitazone and rosiglitazone increased LDL-particle diameter, the increase was greater with pioglitazone. ${ }^{27}$ In another trial, ${ }^{28}$ a total of 305 patients were enrolled in an open-label study. Patients had been taking stable dosages of rosiglitazone and statins for $>90$ days. At baseline, patients discontinued rosiglitazone and started pioglitazone $30 \mathrm{mg} /$ day, but continued statins and other lipid-altering therapies. In addition to the improved shift from smaller, denser to larger LDL particles with pioglitazone, the number of LDL particles decreased. Because statin doses remained stable from baseline, these effects can be attributed to pioglitazone alone. Improvements in LDL diameter and number, as seen in this trial, may have potential cardiovascular benefits.

Use of pioglitazone with statins offers a therapeutic option for diabetic dyslipidemia, particularly for improving triglycerides and for potentially reducing cardiovascular disease risk. In this regard, the completed PROACTIVE study has demonstrated that pioglitazone reduces the composite outcome of nonfatal myocardial infarction, stroke, and allcause mortality in high-risk patients with type 2 diabetes and preexisting macrovascular disease. ${ }^{10}$

Measurement of carotid intima-media thickness (CIMT) has been validated as a measure of atherosclerosis and as a predictor of future cardiovascular events. In Davidson et al's study, the beneficial effect of pioglitazone on HDL cholesterol at 24 weeks predicted its beneficial effect for reducing CIMT progression at 72 weeks. Changes in HDL cholesterol at 24 weeks, irrespective of treatment, predicted less progression of CIMT at 72 weeks. These results suggest that suppression of atherosclerosis with pioglitazone therapy is linked to its ability to raise HDL cholesterol. ${ }^{29}$

A study compared the effects of pioglitazone or rosiglitazone added to glimepiride on a range of lipid parameters, focusing on lipoprotein(a) (Lp[a]) and homocysteine in patients with type 2 diabetes mellitus and metabolic syndrome. The combinations of glimepiride with pioglitazone and glimepiride with rosiglitazone produced significant improvements in measures of glycemic control, plasma lipids, and homocysteinemia. One year of treatment with the pioglitazone combination was associated with significantly reduced plasma $\mathrm{Lp}$ (a) levels compared with the rosiglitazone. ${ }^{30}$

\section{Pioglitazone and inflammation}

Low-grade inflammation is a pathogenic factor for atherosclerosis. High-sensitivity C-reactive protein (hs-CRP), matrix metalloproteinase (MMP)-9, and plasminogen 
activator inhibitor 1 are markers of inflammation. Statins and PPAR $\gamma$ agonists lower inflammatory markers and reduce cardiovascular disease in type 2 diabetes. In a 12-week, prospective, double-blind trial, ${ }^{31} 125$ subjects were randomized to simvastatin or pioglitazone plus placebo or a simvastatin/ pioglitazone combination. At 12 weeks, pioglitazone and simvastatin monotherapies significantly reduced hs-CRP, and the combination regimen had an additive effect. For subgroups, the difference between monotherapy and combination therapy was only significant for simvastatin versus simvastatin plus pioglitazone in patients without metabolic syndrome. Homeostasis model assessment (a measure of insulin resistance) decreased in those receiving pioglitazone, and the correlation between changes in homeostasis model assessment and hs-CRP was significant ( $r=0.43 P<0.05$ ). Plasminogen activator inhibitor 1 decreased significantly in the pioglitazone groups only, and MMP-9 was also significantly lowered in the pioglitazone groups. Pioglitazone, probably by reducing insulin resistance, has additive antiinflammatory effects to simvastatin in nondiabetic subjects with cardiovascular disease and high hs-CRP.

These observational results, obtained from a nonselected patient population under daily routine conditions, confirm the benefits of pioglitazone treatment on blood glucose, lipid metabolism, and blood pressure. The results show that pioglitazone treatment improves chronic vascular inflammation, which may be associated with reduced cardiovascular risk. ${ }^{32}$

Forst et al investigated MMP-9 levels and inflammatory markers during pioglitazone treatment in type 2 diabetic patients with cardiovascular disease. ${ }^{33}$ In their study, during pioglitazone treatment, a 12\% reduction in MMP-9 and an $18 \%$ reduction in hs-CRP levels ( $P<0.05$ for both) could be observed already after 3 days. Monocyte chemotactic protein 1 (MCP-1) levels were reduced by $14 \%$ after 10 days of treatment $(P<0.0001)$. At the end of the study, these parameters were significantly lower in the pioglitazone group compared to the placebo group.

\section{Pioglitazone and myocardial ischemia/reperfusion injury}

In some recent experimental studies using a rat model, treatment with pioglitazone before myocardial ischemia/ reperfusion reduced myocardial infarct size by attenuating reperfusion injury. ${ }^{34,35}$

Ye et al showed the myocardial protective effect of pioglitazone is inducible nitric oxide synthase-independent and may be only partially dependent on endothelial nitric oxide synthase. ${ }^{36}$ In Wang et al, pretreatment with the PPAR $\gamma$ agonist pioglitazone markedly increased the level of phosphorylated extracellular signal-regulated kinase (ERK)-1/2 in rat hearts. PD98059 reversed the cardioprotection exerted by pioglitazone. Increased levels of cyclooxygenase (COX)-2 by pioglitazone were significantly reduced by PD98059. These results imply that pioglitazone decreases apoptosis induced by ischemia/reperfusion injury through the ERK1/2 signaling pathway using COX-2 as the downstream target. ${ }^{37}$

In conclusion, pioglitazone is still an interesting drug. Pioglitazone can still reduce adverse cardiovascular outcomes. Of course, there are a number of critical side effects, such as bone fractures and increased water retention associated with heart failure. In an epidemiological analysis of 66,696 type 2 diabetic subjects followed from 1994 to 2005 in the UK, 6\% used TZDs, with a dose effect on total, hip, and wrist fractures. ${ }^{38}$ The data concerning bladder cancer are unclear. The problem is also that one should select patients for the use of pioglitazone; it is not the drug for everyone. It is certainly the relatively best drug in patients already presenting with cardiovascular disease such as stroke, myocardial infarction, and acute coronary syndrome.

\section{Disclosure}

The authors report no conflicts of interest in this work.

\section{References}

1. Cariou B, Charbonnel B, Staels B. Thiazolidinediones and PPAR $\gamma$ agonists: time for a reassessment. Trends Endocrinol Metab. 2012;23(5): 205-215.

2. Kung J, Henry RR. Thiazolidinedione safety. Expert Opin Drug Saf. 2012;11(4):565-579.

3. Home PD, Pocock SJ, Beck-Nielsen H, et al. Rosiglitazone evaluated for cardiovascular outcomes in oral agent combination therapy for type 2 diabetes (RECORD): a multicentre, randomised, open-label trial. Lancet. 2009;373(9681):2125-2135.

4. Lincoff AM, Wolski K, Nicholls SJ, Nissen SE. Pioglitazone and risk of cardiovascular events in patients with type 2 diabetes mellitus: a meta-analysis of randomized trials. JAMA. 2007;298(10): $1180-1188$.

5. Watkins PB, Whitcomb RW. Hepatic dysfunction associated with troglitazone. N Engl J Med. 1998;338(13):916-917.

6. Nissen SE, Wolski K. Effect of rosiglitazone on the risk of myocardial infarction and death from cardiovascular causes. $N$ Engl $J$ Med. 2007;356(24):2457-2471.

7. Lago RM, Singh PP, Nesto RW. Congestive heart failure and cardiovascular death in patients with prediabetes and type 2 diabetes given thiazolidinediones: a meta-analysis of randomized clinical trials. Lancet. 2007;370(9593):1129-1136.

8. Dorkhan M, Dencker M, Stagmo M, Groop L. Effect of pioglitazone versus insulin glargine on cardiac size, function and measures of fluid retention in patients with type 2 diabetes. Cardiovasc Diabetol. 2009;8: $15-20$.

9. Singh S, Loke Y, Furberg C. Long-term risk of cardiovascular events with rosiglitazone: a meta-analysis. JAMA. 2007;298(10):1189-1195. 
10. Dormandy JA, Charbonnel B, Eckland DJA, et al. Secondary prevention of macrovascular events in patients with type 2 diabetes in the PROACTIVE Study (Prospective Pioglitazone Clinical Trial in Macrovascular Events): a randomised controlled trial. Lancet. 2005;366(9493):1279-1289.

11. Fanarow GC. Approach to the management of diabetic patients with heart failure: role of thiazolidinediones. Am Heart J. 2004;148(4): 551-558.

12. Masoudi FA, Inzucchi SE, Wang Y, Havranek EP, Foody JM, Krumholz HM. Thiazolidinediones, metformin and outcomes in older patients with diabetes and heart failure. An observational study. Circulation. 2005;111(5):583-590.

13. Haffner SM, Lehto S, Ronnemaa T, Pyorala K, Laakso M. Mortality from coronary heart disease in subjects with type 2 diabetes and in nondiabetic subjects with and without prior myocardial infarction. N Engl J Med. 1998;339(4):229-234.

14. Brener SJ, Lytle BW, Casserly IP, Topol EJ, Lauer MS. Propensity analysis of long-term survival after surgical or percutaneous revascularization in patients with multivessel coronary disease and high-risk features. Circulation. 2004;109(19):2290-2295.

15. Scholte AJ, Schuijf JD, Kharagjitsingh AV, et al. Prevalence of coronary artery disease and plaque morphology assessed by multi-slice computed tomography coronary angiography and calcium scoring in asymptomatic patients with type 2 diabetes. Heart. 2008;94(3):290-295.

16. Waller BF, Palumbo PJ, Lie JT, Roberts WC. Status of the coronary arteries at necropsy in diabetes mellitus with onset after age 30 years: analysis of 229 diabetic patients with and without clinical evidence of coronary heart disease and comparison to 183 control subjects. $\mathrm{Am} J$ Med. 1980;69(4):498-506.

17. Gu K, Cowie CC, Harris MI. Diabetes and decline in heart disease mortality in US adults. JAMA. 1999;281(14):1291-1297.

18. Nissen SE, Nicholls SJ, Wolski K, et al. Comparison of pioglitazone vs glimepiride on progression of coronary atherosclerosis in patients with type 2 diabetes: the PERISCOPE randomized controlled trial. JAMA. 2008;299(13):1561-1573.

19. Kuusisto J, Mykkänen L, Pyörälä K, Laakso M. Hyperinsulinemic microalbuminuria: a new risk indicator for coronary heart disease. Circulation. 1995;91(3):831-837.

20. Eastman RC, Keen H. The impact of cardiovascular disease on people with diabetes: the potential for prevention. Lancet. 1997;350 Suppl 1: SI29-SI32.

21. Mattock MB, Morrish NJ, Viberti G, Keen H, Fitzgerald AP, Jackson G. Prospective study of microalbuminuria as predictor of mortality in NIDDM. Diabetes. 1992;41(6):736-741.

22. Hanefeld M, Brunetti P, Schernthaner GH, Matthews DR, Charbonnel BH. One-year glycemic control with a sulfonylurea plus pioglitazone versus a sulfonylurea plus metformin in patients with type 2 diabetes. Diabetes Care. 2004;27(1):141-147.

23. Agrawal B, Wolf K, Berger A, Luft FC. Effect of antihypertensive treatment on qualitative estimates of microalbuminuria. J Hum Hypertens. 1996;10(8):551-555.

24. Nakamura T, Ushiyama C, Osada S, Hara M, Shimada N, Koide H. Pioglitazone reduces urinary podocyte excretion in type 2 diabetes patients with microalbuminuria. Metabolism. 2001;50(10):1193-1196.
25. Koskinen P, Manttari M, Manninen V, Huttunen JK, Heinonen OP, Frick MH. Coronary heart disease incidence in NIDDM patients in the Helsinki Heart Study. Diabetes Care. 1992;15(7):820-825.

26. Haffner SM. Dyslipidemia management in adults with diabetes. Diabetes Care. 2004;27 Suppl 1:S68-S71.

27. Goldberg RB, Kendall DM, Deeg MA, et al. A comparison of lipid and glycemic effects of pioglitazone and rosiglitazone in patients with type 2 diabetes and dyslipidemia. Diabetes Care. 2005;28(7): 1547-1554.

28. Berhanu P, Kipnes MS, Khan MA, et al. Effects of pioglitazone on lipid and lipoprotein profiles in patients with type 2 diabetes and dyslipidaemia after treatment conversion from rosiglitazone while continuing stable statin therapy. Diab Vasc Dis Res. 2006;3(1):39-44.

29. Davidson M, Meyer PM, Haffner S, et al. Increased high-density lipoprotein cholesterol predicts the pioglitazone-mediated reduction of carotid intima-media thickness progression in patients with type 2 diabetes mellitus. Circulation. 2008;117(16):2123-2130.

30. Derosa G, Cicero AF, D'Angelo A, et al. Effects of 1 year of treatment with pioglitazone or rosiglitazone added to glimepiride on lipoprotein (a) and homocysteine concentrations in patients with type 2 diabetes mellitus and metabolic syndrome: a multicenter, randomized, doubleblind, controlled clinical trial. Clin Ther. 2006;28(5):679-688.

31. Hanefeld M, Marx N, Pfützner A, et al. Anti-inflammatory effects of pioglitazone and/or simvastatin in high cardiovascular risk patients with elevated high sensitivity C-reactive protein: the PIOSTAT Study. J Am Coll Cardiol. 2007;49(3):290-297.

32. Karagiannis E, Pfützner A, Forst T, et al. The IRIS V study: pioglitazone improves systemic chronic inflammation in patients with type 2 diabetes under daily routine conditions. Diabetes Technol Ther. 2008;10(3) 206-212.

33. Forst T, Karagiannis E, Lübben G, et al. Pleiotrophic and antiinflammatory effects of pioglitazone precede the metabolic activity in type 2 diabetic patients with coronary artery disease. Atherosclerosis. 2008;197(1):311-317.

34. Honda T, Kaikita K, Tsujita K, et al. Pioglitazone, a peroxisome proliferator-activated receptor-gamma agonist, attenuates myocardial ischemia-reperfusion injury in mice with metabolic disorders. $J \mathrm{Mol}$ Cell Cardiol. 2008;44(5):915-926.

35. Li J, Lang MJ, Mao XB, Tian L, Feng YB. Antiapoptosis and mitochondrial effect of pioglitazone preconditioning in the ischemic/reperfused heart of rat. Cardiovasc Drugs Ther. 2008;22(4):283-291.

36. Ye Y, Lin Y, Manickavasagam S, Perez-Polo JR, Tieu BC, Birnbaum Y. Pioglitazone protects the myocardium against ischemiareperfusion injury in eNOS and iNOS knockout mice. Am J Physiol Heart Circ Physiol. 2008;295(6):H2436-H2446.

37. Wang H, Zhu QW, Ye P, et al. Pioglitazone attenuates myocardial ischemia-reperfusion injury via up-regulation of ERK and COX-2. Biosci Trends. 2012;6(6):325-332.

38. Meier C, Kraenzlin ME, Bodmer M, Jick SS, Jick H, Meier CR. Use of thiazolidinediones and fracture risk. Arch Intern Med. 2008; 168(8):820-825.
Vascular Health and Risk Management

\section{Publish your work in this journal}

Vascular Health and Risk Management is an international, peerreviewed journal of therapeutics and risk management, focusing on concise rapid reporting of clinical studies on the processes involved in the maintenance of vascular health; the monitoring, prevention and treatment of vascular disease and its sequelae; and the involvement of

\section{Dovepress}

metabolic disorders, particularly diabetes. This journal is indexed on PubMed Central and MedLine. The manuscript management system is completely online and includes a very quick and fair peer-review system, which is all easy to use. Visit http://www.dovepress.com/ testimonials.php to read real quotes from published authors. 